\title{
FLASHES HISTÓRICOS SOBRE EL PROSTATSMO Y LA RETENCIÓN DE ORINA.
}

\author{
A taúlfo Saíz Carrero.
}

Especialista Senior. Jubilado Servicio de Urología. Hospital G eneral Universitario G regorio Marañón. M adrid. España

\begin{abstract}
Resumen.- O BJETIVO: Realizar una búsqueda cronológica sobre la sintomatología obstructiva vesical de origen prostático y algunos de sus tra tamientos.

MÉTO DO S/ RESULTADOS: Se han ido recopilando, durante años, da tos de libros y revistas que hacen referencia al tema objeto del estudio, presentados de manera que puedan ser consultados cronológicamente. De más de 100 referencias se han seleccionado las sesenta más relevantes a nuestro parecer.

CON CLUSIONES: Muchas ideas han surgido sobre este síndrome y la manera de solucionarlo, con variaciones según la época. Para la mayoría de los urólogos, la principal complicación opera toria ha sido siempre la hemorragia, lo que ha motivado el desarrollo de gran cantidad de técnicas para combatirla.
\end{abstract}

A taúlfo Saíz Carrero

C/ Sanchez Pacheco, 62 - $3 \stackrel{0}{2}$

28002 Madrid. (España)

Trabajo recibido: 1 de diciembre 2004
La otra gran complicación, la infección, fue motivó de que las maniobras quirúrgicas fueran realizadas, en un principio, con mucha rapidez y pequeñas incisiones. Eran necesarios, además, gruesos drenajes.

Palabras clave: Historia de la Urología. Prostatismo. Retención de orina. Bosquejo histórico.

Summary.- O BJEC TIVES: To perform a chronological search a bout symptoms of bladder outlet obstruction of prostatic origin and some of its treatments.

METHODS/ RESULTS: O ver the years we have been collecting data about the topic from books and journals; we present them in such a way that they can be consulted chronologically.

From more than 100 references we have selected the 60 most relevant to our judgment.

CON CLUSIO N S: M any ideas about the syndrome and the way to solve it have arisen, which vary from one time to another. For most urologists, the main operative complication has always been bleeding, leading to the development of a great number of techniques to avoid it Infection, the other great complication, prompted that initially surgical maneuvers were performed very fastly and with the small incisions. In addition, big drainages were necessary.

Keyw ords: History of urology. Symptoms of BPH. Urinary retention. Historical review. 
“...Porque hurtar en cosa de ciencia, y de letras, no solamente no es vicio, mas es muy grande virtud, y honra, porque es señal que el hombre ha estudiado, y trabajado, para aprovecharse él, y beneficiar a otros. $Y$ esto es cierto lo hacen muchos de los que han escrito, y hacen el día de hoy algunos, con 10 cual se honran mucho. Todo lo cual, a mi parecer, está en su lugar; pues les cuesta su trabajo, y ponen en ello su diligencia, e industria..." (Miguel de Leriza, 1665).

\section{0 - 1200 a. C. Táblillas Mesopotámicas} Primeras referencias a la sintomatología prostática, manifestadas por los dos grupos encargados de la medicina: Asu y Asipu. (8)

\section{$\sim 3000$ a. C. Sucruta}

En el Ayur-Veda se mencionan métodos para combatir la retención de orina así como la realización de la talla. (11)

\section{-930 a. C. Eclesiastés}

Los síntomas del prostatismo, según Everard Home (uno de los fundadores del Real Colegio de Cirujanos

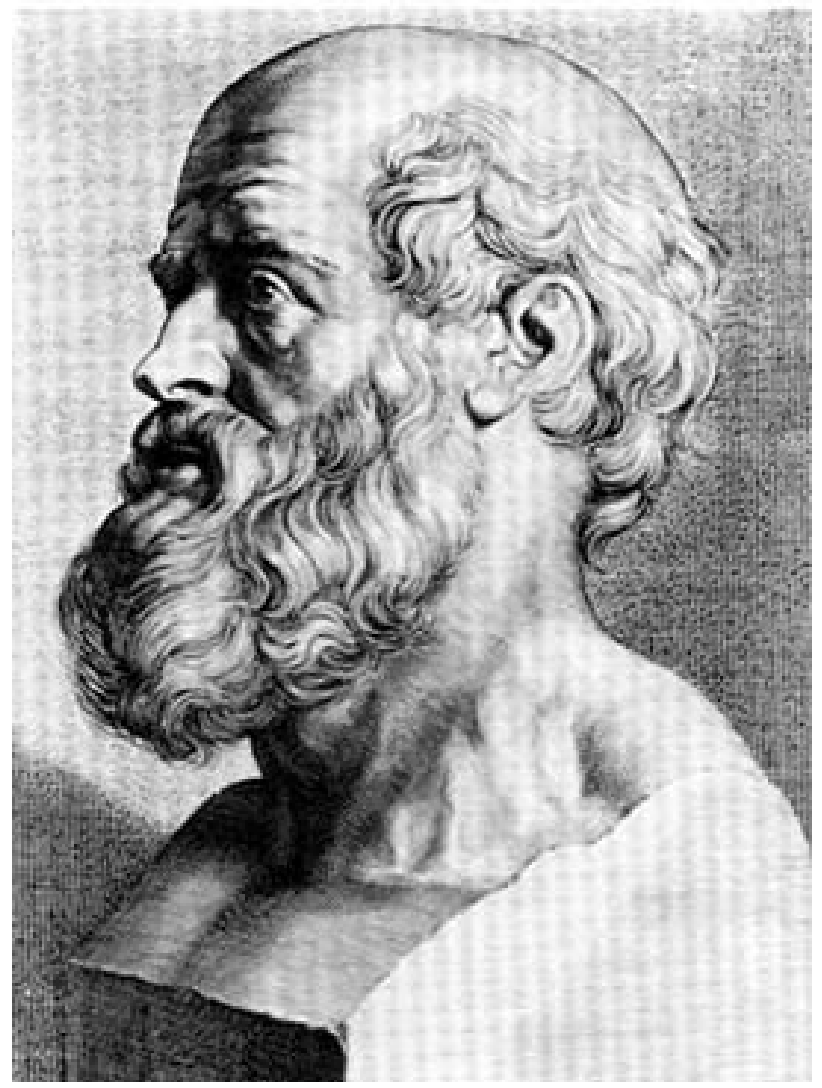

FIG URA 1. Hipócrates. Rémora de la cirugía vesical. de Londres), se reflejan en la Biblia. Este ingenioso autor dijo que los síntomas que el aumento de la próstata produce, tan frecuentes en la vejez, son mencionados en la bonita descripción del envejecimiento que se lee en el Antiguo Testamento. En el libro de Eclesiastés, capítulo 12, versículo 6, está escrito: "el cántaro está roto junto a la fuente, y la rueda rota sobre el pozo"; expresivo de los dos efectos principales de esta enfermedad, la salida involuntaria de la orina, y la interrupción total de ella. (12)

\section{0 a. C. Hipócrates}

Hipócrates: en su juramento expone: “...no practicaré jamás la operación de talla...".

Uno de sus aforismos, decía: "La rotura de la vejiga, del cerebro, del corazón, del diafragma, de alguno de los intestinos delgados, del estómago y del hígado, es mortal". (Sección 6ă, aforismo no 18). Por todo ello no a consejaba a sus discípulos operar la vejiga. $(8,36)$

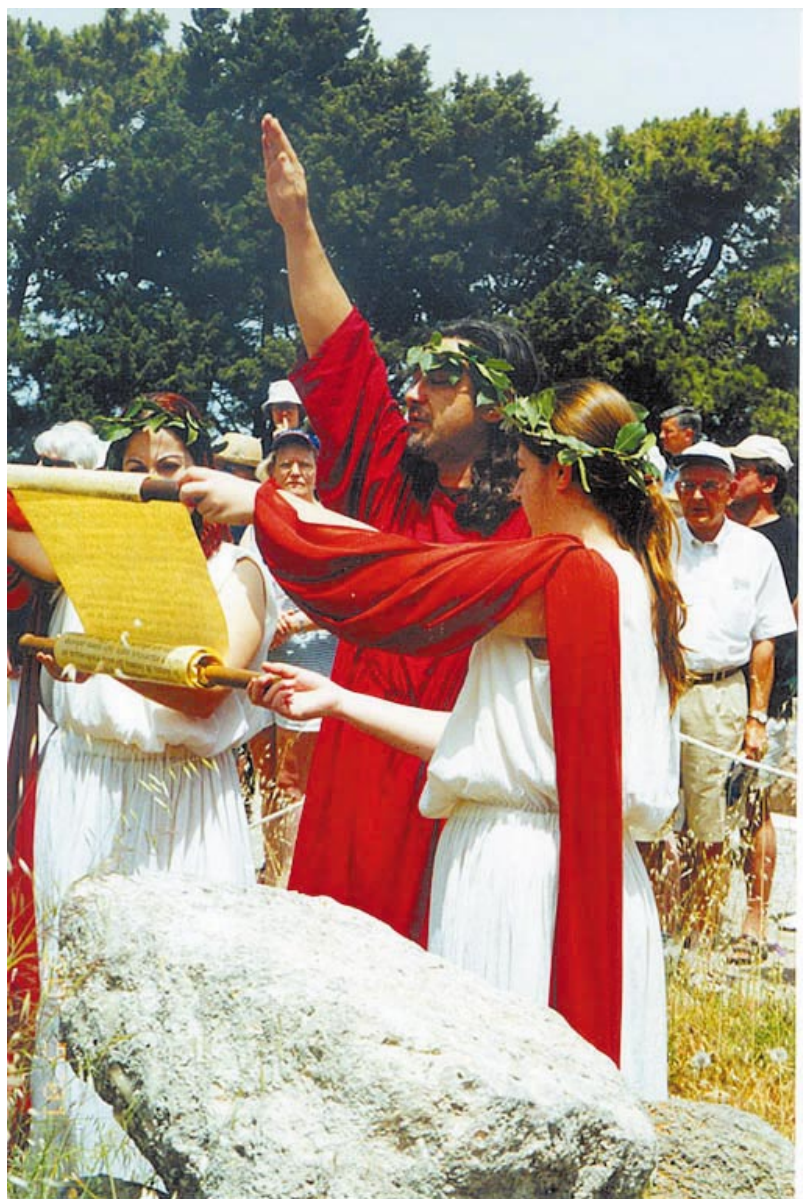

FIG URA 2. Juramento hipocrático en la isla de Kos, en la actualidad. 
En otros aforismos comenta: "Si la vejiga de la orina está dura y dolorosa, es síntoma muy grave mortal, sobre todo cuando se agrega una fiebre continua" y "una supresión de orina con pesadez en el hipogastrio, anuncia una estranguria cercana, siendo esta más larga en el hombre de cierta edad que en el joven". (14) (Figuras 1 y 2)

\section{$\sim 280$ a. C. Herófilo de Calcedonia}

Posiblemente, fue de los primeros en hacer disecciones en cadáveres humanos. Lo habitual era hacerlas en a nimales. Realizó la primera descripción de la próstata. Le dio el nombre de parastate del que deriva el actual. $(11,12,52)$

\section{$\sim 270$ a. C. Epicuro}

Filósofo griego que elaboró una original doctrina sobre el placer. Murió después de 14 días de padecer retención de orina (Séneca, cartas). (52)

\section{$\sim 250$ a. C. Ammonius}

Llamado El Litotomo porque perfeccionó la talla vesical, aunque la realizaba con reserva y cautela. (11)

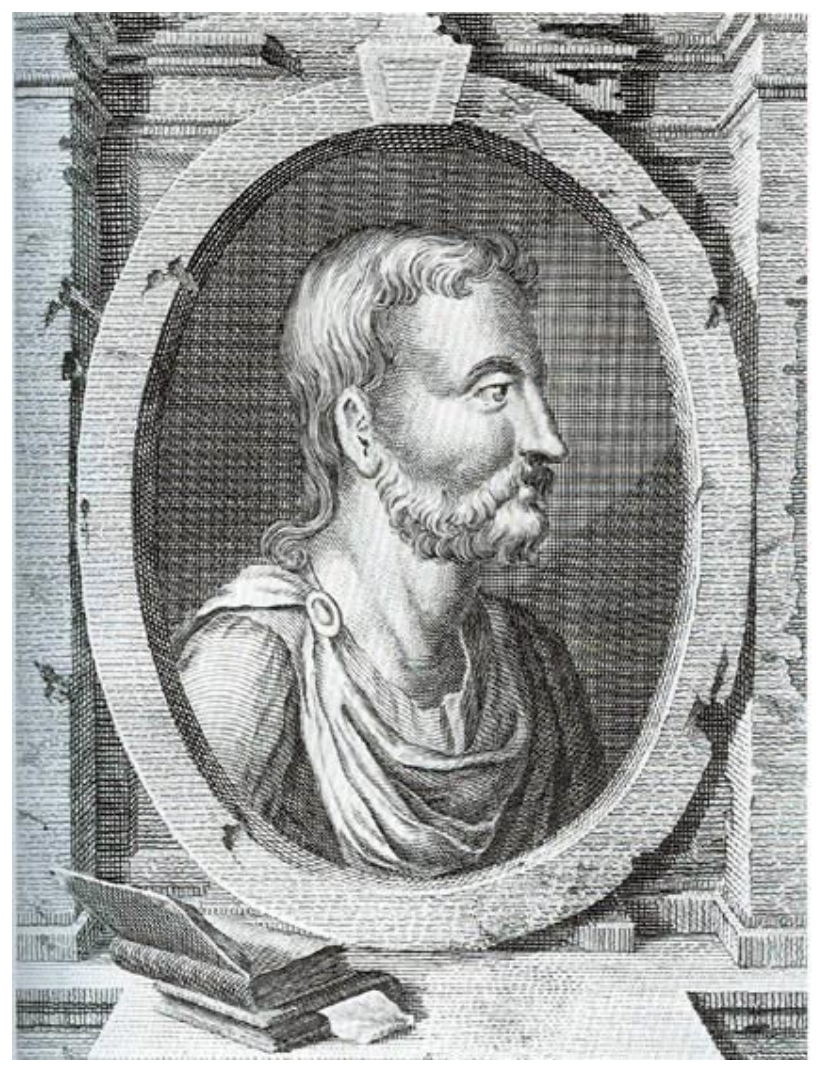

FIG URA 3. Cornelio Celso gran conocedor de la técnicas quirúrgicas de su época.

\section{$\sim 23$ a. C. Cornelio Celso}

A unque no fue médico conocía bien los procedimientos quirúrgicos. En su gran obra De Re Medica (escrita en latín) realiza una admirable descripción de la operación de la talla (apparatus parvus).

Muy pocos se habían atrevido antes a abordar la vejiga, obedeciendo los consejos de Hipócrates. La técnica que describió fue empleada hasta el siglo XVII. $(8,11)$ (Figura 3)

\section{$\sim 128$ d. C. Rufus de Éfeso}

Al parecer fue el primero que describió la próstata como una glándula y sus relaciones con los deferentes, en su obra De vesicae renunque affectibus. Fue un competente cirujano. $(8,12)$

\section{1 d. C. Galeno}

Pionero en el estudio de las carnosidades y callosidades del cuello de la vejiga que obstruían la salida de la orina y que podían ser destruidas por medio de un catéter. Luego Amatus Lusitanus y Juan de Vigo hablan también de cateterismos forzados. $(12,13)$

\section{-355 d. C. Oribasio de Pérgamo}

Propuso cortar por vía perineal la "induración del cuello de la vejiga", cuando era imposible el sondaje. Era mejor tener una fístula que morir de retención urinaria. $(5,8)$

\section{Rhazès}

En su libro Continens, describe la talla según el procedimiento de Pablo de Egina (625-690) (apparatus parvus). (11)

\section{Abulcasis}

Practicó la talla con técnica igual que la de Celso y Pablo de Egina, pero con más perfección. Sin embargo, decía en su libro Altasrif: "no utilicéis la cirugía más que cuando hayan fracasado los medios suaves y emolientes". (11)

\section{Avenzoar}

Director de un hospital de Sevilla. Nunca practicó la talla pues consideraba impúdica cualquier intervención en los órganos genitales del hombre y de la mujer. (11)

\section{Doctores Reina y Ruiz de Medina}

Catedráticos de Salamanca. Realizaron una talla al que más tarde sería San Juan de Sahagún. (11) 


\section{Francisco López de Villalobos}

Fue médico de Fernando el Católico, del Cardenal Cisneros, del Duque de Alba y de Carlos I. En su libro Sumario de la Medicina escribe en poesía los síntomas del prostatismo:

\section{De la destilacion de la urina o estranguria}

Tenerse la urina se llama stranguria, y esto es quando sale goteando y poquita; si no sale nada, llamámosla suria, y si sale a ratos, se llama disuria; y es una la cura en todas escrita: aquesto procede de alguna frialdad, o llaga o calor, o de algún apostema questá en la vexiga, o en su vezindad, 0 está en la virtud, esta enfermedad, o es por humores mayormente flema.(11)

\section{Paré}

Fue el primero en descubrir una operación bien sistematizada en la que mediante una sonda especial trataba de destruir transuretralmente el tejido que a su juicio obstruía el cuello de la vejiga. $(5,12,54)$

\section{Vesalio}

La próstata, redescubierta en esta época por $\mathrm{N}$ iccolo Massa (médico veneciano que murió en 1563), fue

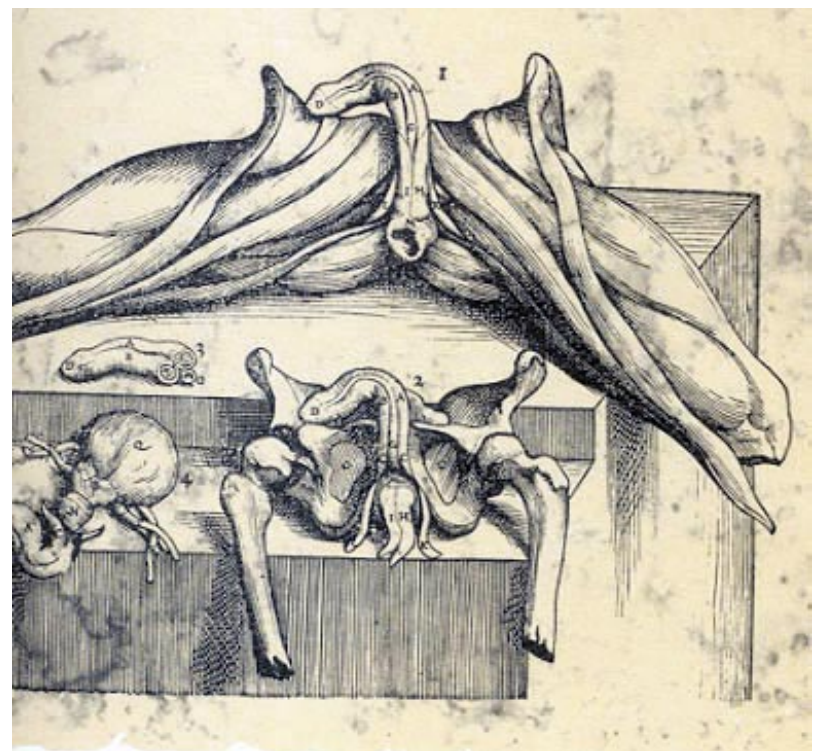

FIG URA 4. Parte inferior de la lámina 41 del libro de Vesalio:"De Humanis Corporis Fabrica". bien estudiada por Vesalio, a unque incluía en bloque las vesículas seminales. Por primera vez hay una representación gráfica de ella en su libro De Humanis Corpore Fabrica. (30) (Figura 4)

\section{Andrés Laguna}

En su Methodus cognoscendi extirpandique excrescentes in vesicae collo carunculas, escribe: "hay excrecencias o carnosidades que asientan en la uretra cerca del cuello vesical, que dificultan la micción y hasta llegan a suprimirla, produciendo retención" Sondaba con candelillas y la maniobra no era suficiente usaba el catéter de plomo, y si fallaba, los de plata o de oro. Se producían, frecuentemente, hemorragias grandes. $(14,35)$

\section{Juan Valverde de Amusco}

Con su obra Historia de la composición del cuerpo humano despertó el interés por la Anatomía en España. Fue gran admirador de Vesalio pero al que rectificó en algunos puntos. (11)

\section{Pierre Franco}

De forma circunstancial o accidental, fue el primero que abordó la vejiga por vía suprapúbica (apparatus altus) en un niño de dos años, que sanó. Sin embargo, no volvió a repetir esta vía de acceso. $(36,40)$

\section{Francisco Díaz}

En su célebre libro: Tratado nuevamente impreso de todas las enfermedades de los riñones, vejiga y carnosidades de la verga, y orina, describe la operación de talla con una técnica análoga a la de Celso pero modificada, pues hacía la incisión perineal lateralizada. También modificó los instrumentos para realizarla. Comenta que: "algunos llegan atrevidamente a hacer esta obra, y en estos se había de poner grande castigo y remediar que no quedase en poder de idiotas y bajos hombres que ni saben ni quieren saber... y como los más son viandantes y hoy aquí y mañana allí, no esperan suceso malo, más que coger el dinero y volar".

Sobre las carnosidades opina que también se pueden formar en el cuello de la vejiga produciendo retención. (18)

Según Alfonso de la Peña, Francisco Díaz fue el creador del primer resector o uretrotomo, consistente en un trócar curvo con un mandril punzante en forma de pirámide. $(8,11,14)$ 


\section{Jean Riolano (Junior)}

Practicó la incisión del cuello de la vejiga a través del periné en casos de retención. Fue el primero en sugerir que la vejiga podía estar obstruida por un tumor de la próstata. Casi cien años más tarde, Santorini, reconoce esta misma circunstancia. (52)

\section{Covillarc}

Fue el primero en extraer un lóbulo medio después de una talla perineal. (12)

\section{Régnier de Graaf}

A natómico holandés que precisó la naturaleza glandular de la próstata, en su obra De virorum organis generationi inserventibus. (52)

\section{John Douglas}

En este año, restaura la talla suprapúbica, casi olvidada durante más de un siglo. La realizó de forma programada. La siguió y la perfeccionó su discípulo Cheselden, hábil cirujano que llegó a realizar perfectas litotomías en cincuenta y cuatro segundos. (12)

\section{Blas Beaumont}

Profesor del Hospital General de Madrid en su obra Exercitaciónes anatómicas trata de la talla a la que llamaba "lithotomía". (11)

\section{Martín Martínez}

Trabajó en el mismo hospital que Beaumont. En su libro Medicina scéptica y cirugía moderna descubre una técnica de talla casi análoga a la de ese cirujano. Escribe: "los vicios de la uretra casi siempre son producidos por el sigilo venenoso... el cuello de la vexiga se comprime por las Próstatas endurecidas; la orina sale con dificultades, y forma un caño muy delgado..." (11)

En su A natomía completa del hombre (1728) describe con gran precisión el aparato génito urinario. Fue el texto de anatomía más importante de la primera mitad de esa centuria. (53)

\section{Morgagni}

En su libro Sedibus et causis morborun publicó 800 protocolos de autopsias. Identificó como entidad la hipertrofia de la próstata. Se le considera el fundador de la Anatomía Patológica. (8)

\section{Francisco Villaverde}

En su: "O peraciones de cirugía, según las más selec- tas doctrinas de antiguos y modernos", menciona la talla hipogástrica pero la considera peligrosa. Manifiesta su preferencia por el método simplificado de talla perineal que ya realizaba el cirujano español Francisco Canibel. Su obra es muy completa en la descripción de técnicas de talla. (11)

\section{Juan Naval}

Escribe en España el único tratado de urología de esa centuria. En él recoge y enriquece todo saber urológico precedente: Tratado médico quirúrgico de las enfermedades de las vías de orina. En su segundo tomo hace un amplio estudio del síndrome de retención urinaria, con mención especial a las afecciones prostáticas: "la hinchazón y endurecimiento escirroso de la próstata es otra enfermedad muy común a los ancianos, y a los que han padecido muchas gonococias". Describe cómo solucionarlo con el sondaje o la punción de la vejiga. En otra sección se ocupa de la talla, aunque algo superficialmente. (53)

\section{John Hunter}

Comunicó a la Sociedad Médica Real de Londres del descubrimiento del lóbulo medio como responsable de la mayoría de las enfermedades que obstruían las vías urinarias. Su yerno, Everard Home, publicó en 1810 un tratado en donde se veía, por vez primera, un dibujo que mostraba una obstrucción urinaria por un lóbulo medio. Lo había plagiado de su suegro. También describió los tres lóbulos prostáticos. $(5,54)$

\section{Blizard}

Fue el primero en intentar una sección del cuello vesical por vía abierta. (5)

\section{J. Zulema Amussat}

Como complemento de una cistolitotomía por vía hipogástrica, escindió con tijera un lóbulo medio. Esta vía estuvo, otra vez, abandonada casi por completo hasta 1885. Aún quedaba el recuerdo del aforismo de Hipócrates. $(10,40,54)$

\section{Guthrie}

Hizo una sonda con una cuchilla oculta que podía ser proyectada para seccionar la parte del cuello vesical que producía la obstrucción. Describió una enfermedad con los síntomas de la hipertrofia pero con la glándula de tamaño normal. Es la primera descripción de la enfermedad del cuello vesical. (5)

Poco después Civiale y Mercier diseñaron 
unos instrumentos parecidos. Según ellos, fueron hechos antes de los de Guthrie. Mercier inició el término "hipertrofia prostática" (54)

\section{Fergusson}

Mostró una pieza de hipertrofia prostática que había extirpado después de un cálculo vesical por vía perineal. Le siguió Kuchler que matizó y perfeccionó la operación, realizándolas en cadáveres. (12)

Dos años antes Morton ya había introducido la anestesia general.

\section{Henry Thompson}

Era el más famoso urólogo de aquella época. Empleó muy a menudo el drenaje a través del periné. Pero no creía en la efectividad de la operación. "La atonía que sufría el músculo de la vejiga impediría luego la micción". Lo mismo opinaban en A lemania August Socin y en Francia Félix Guyón en 1880. (40,54) (Figura 5)

\section{Paget}

Dijo que el agrandamiento de la próstata podía ser enucleado en su totalidad, y esta idea se fue extendiendo gracias a los estudios anatómicos y al mayor número de operaciones que se hacían. (8)

\section{Bottini}

Inventó un divulsor con cauterio eléctrico y accionado con una cremallera, para realizar una incisión sobre el cuello de la vejiga y el lóbulo medio de la próstata, y así eliminar el obstáculo. $(5,38)$ (Figura 6)

\section{Billroth}

Usando el método descrito por Kuchler, realizó dos prostatecto mías en vivos pero no fueron completas. (10)

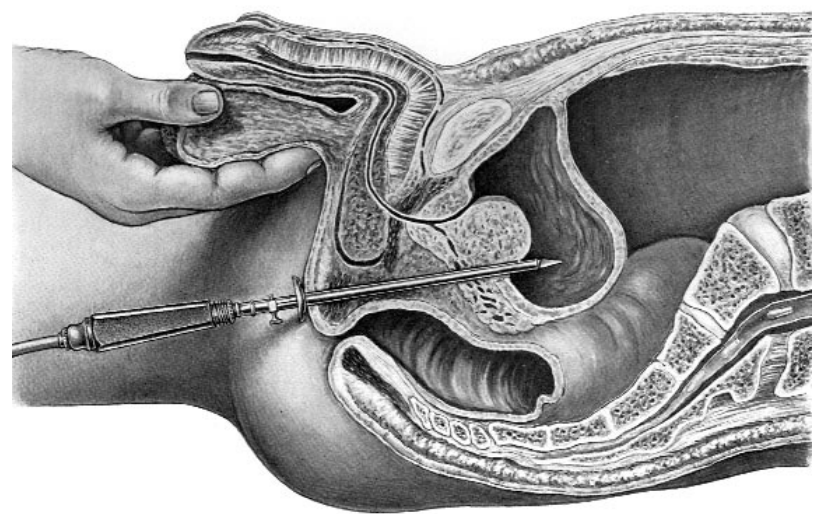

FIG URA 5. Punción vesical por vía perineal.
Este mismo año Lister publica sus dos conocidos artículos sobre asepsia.

\section{Von Dittel}

Realizó, planeada, la primera adenomectomía parcial. Con un lazo extrajo un lóbulo medio, en un enfermo que ya tenía una cistostomía suprapúbica previa. Los buenos resultados de la operación realizada por A mussat habían animado, también, a otros a hacerla. Cuando encontraban un lóbulo medio lo ligaban y seccionaban con tijeras, bisturí o termocauterio. Le siguieron Trendelenburg (1886), Schmidt (1888), Kümmel (1889) McGill, Mayo, Robson, Atkinson, Teale, Guyon (1889) y Watson. $(8,40)$

Este mismo año, Tillaux en su "Tratado de Cirugía Clínica": opinaba lo siguiente:

"En los casos de retención de orina por hipertrofia de la próstata, vaciar la vejiga por medio de una sonda apropiada, y si esto no es posible, puncionar la vejiga por encima del pubis. Ensayar nuevamente el cateterismo. Si después de repetidos ensayos no podemos realizarlo (lo cual es muy excepcional), sustitúyase la punción suprapúbica por la talla hipogástrica. Explórese a tentamente la región del cuello y extírpese el lóbulo medio de la próstata si parece ser la causa de los accidentes, y sobre todo si está pediculado. Si ninguna intervención sobre la próstata parece racionalmente posible, establézcase una uretra suprapúbica permanente".

(57) (Figura 7)

\section{Belfield}

El primero que realizó una cistotomía suprapúbica con la finalidad de extraer un lóbulo medio, sin necesidad de que hubiera una derivación previa. Comentaba que a lgunos fallos quirúrgicos eran debidos tanto a las operaciones incompletas, como al estudio insuficiente de la uretra. En 1890 hizo una revisión de las prostatectomías realizadas hasta entonces, y de 133, 88 habían sido hechas por vía suprapúbica y las otras por vía perineal o combinadas. (54)

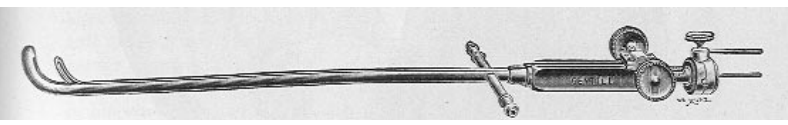

FIG URA 6. Divulsor de Bottini. 


\section{McGill}

Pionero en Inglaterra. Escindió lóbulos medios por talla vesical hipogástrica. Describió tres casos que resultaron un éxito. "... extirpando con tijeras y fórceps la parte aumentada de la próstata..." (10)

\section{Goodfellow}

Realizó la primera adenomectomía perineal completa un mes antes de su compatriota W ishard. A mbos desconocían el trabajo del otro. Le siguieron en su país (Norteamérica) Carpenter, Mac Lean y sobre todo Young (decía Keynes en 1903: "la próstata hace viejos a la mayoría de los hombres pero hizo a Hugh Young").

\section{En Europa: Proust, Gosset y Albarrán.}

Hasta entonces, casi todas las que se hacían eran parciales (lóbulos medios). Las primeras fueron realizadas con incisiones longitudinales del periné. $(11,54)$

\section{White y Ramm}

Recomendaron la castración quirúrgica para curar la hipertrofia prostática, siguiendo la teoría hipotética de A dams, que relacionaba la próstata con la función testicular. Ya había sido practicada por Snitin en 1886

Se usó también la orquiectomía unilateral (Burckhardt) y las inyecciones esclerosantes en el parénquima testicular (Mac Cully). La ligadura del cordón fue propuesta por varios autores entre ellos Albarrán.

Bier en este mismo año ligó las dos arterias iliacas internas con el fin de atrofiar la próstata y su "adenoma". De 11 enfermos, 3 fallecieron. (54)

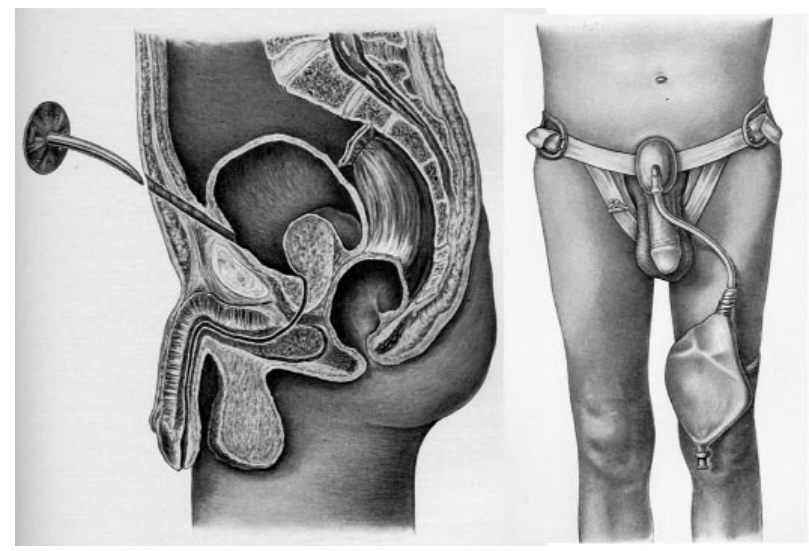

FIG URA 7. Uretra hipogástrica permanente.

$$
\text { 1893. Jores }
$$

0 pinaba que en algunos casos la alteración partía de las glándulas prostáticas periuretrales. Albarrán (1900) y después Motz y Perearnau (1905), recogen esta idea y añaden que toda hipertrofia tenía como origen dichas glándulas. (12)

\section{Fuller}

Fue el primero que realizó, sistemáticamente, adenomectomías totales. Abría la vejiga con una pequeña incisión alta, con el fin de no implicar el espacio prevesical retropubiano. Empujaba por el periné con la otra mano y enucleaba con un dedo. Previamente cortaba la mucosa con tijera. Dejaba un drenaje por el periné. Colocaba un buen tubo suprapúbico. Hacia el año 1905 había ya realizado más de 300 intervenciones.

$\mathbf{N}$ icol y Alexander recomendaban lo contrario: presionar el adenoma por vía transvesical y enuclearlo por vía perineal. $(10,23,54)$

\section{Guiard}

Diseñó un armario para la autocateterización en el domicilio. Pero también había artilugios para poder llevar la sonda y poder sondarse fuera de casa. A principios del S. XIX, antes de extenderse la cirugía, los enfermos prostáticos ingleses eran ingresados 1-2 semanas para enseñarles el sondaje intermitente. La infección y la insuficiencia renal producían un $8 \%$ de muertes. $(5,26)$ (Figura 8).

\section{Ramón Guiteras}

A prendió la técnica de su amigo Fuller (eran compa-

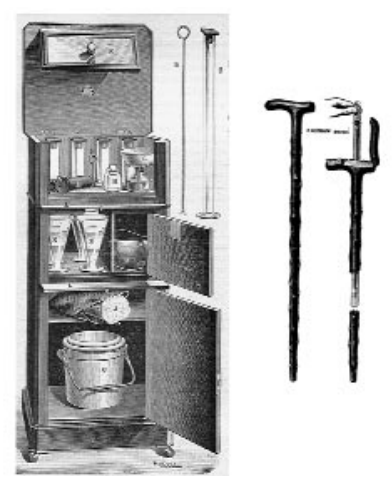

FIG URA 8. Armario para autosondaje y bastón portador de sondas. 
ñeros de boxeo) y la modificó presionando con dos dedos introducidos en el recto. La comunicó en el Congreso Internacional de Medicina que se celebró en París en A gosto de este año. $(10,40,54)$

\section{Freyer}

Durante su estancia en Europa, el Dr. Guiteras visitó a Freyer en su Hospital de Londres y le enseñó la técnica de Fuller. O peró su primer caso en diciembre de 1900.

En 1901 comunicó que era una técnica suya: Realizaba una pequeña apertura en la vejiga (operación "cerrada") y actuaba con gran rapidez (15 minutos). Dejaba un gran drenaje suprapúbico y no colocaba sonda uretral. No empujaba desde el periné, ni dejaba drenaje por esa zona, y cortaba la mucosa periférica al adenoma con una uña. Creía que extirpaba la próstata por completo. Estas ideas fueron refutadas por Mayo, Fuller, Thomson y Wallace.

En 1912 publicó sus 1000 primeros casos. $(10,21$ $23,40,54)$ (Figura 9)

\section{Audry}

Dada la gran mortalidad que existía, ideó este cirujano francés, la intervención en dos tiempos. Primero realizaba una talla vesical en situación alta y colocaba una sonda hipogástrica. A los 15-20 después hacía la adenomectomía.

La mortalidad disminuyó, porque con este proceder, mejoraba la función renal, disminuía la infección y se eliminaban cálculos coincidentes. Al obliterarse el espacio prevesical desaparecían las

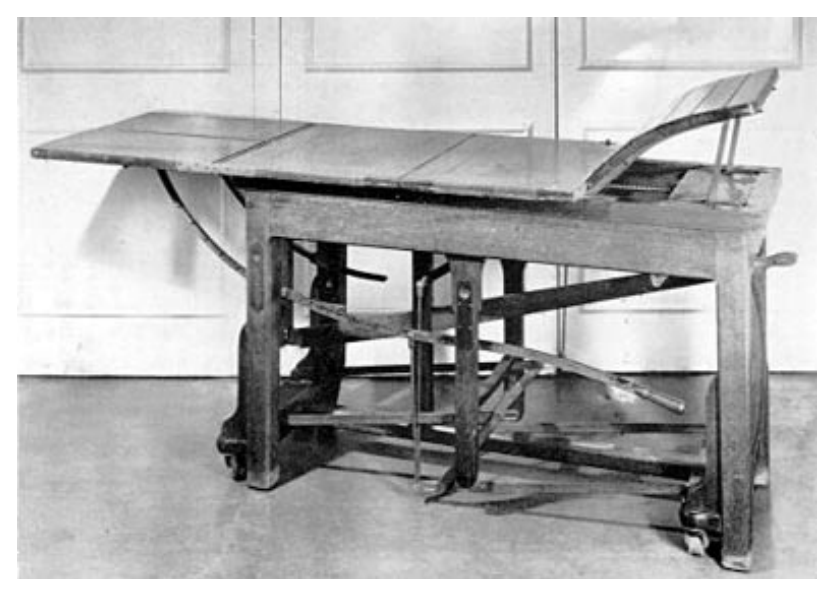

FIG URA 9. M esa en la que operó su primera prostatectomía suprapúbica Sir Peter Freyer. infecciones en esa zona. (6)

Según Gordon A. Nicoll y colaboradores, esta mortalidad fue descendiendo al cabo de los años de la manera siguiente: Los pioneros: Belfield, MCGill y Fuller, alrededor del $25 \%$; Freyer el $9 \%$ y Harris el $2,5 \%$. (46)

\section{Howard Lilienthal}

Fue uno de los primeros en realizar en USA, la operación en dos tiempos.

André y Carrier este mismo año y Pauchet en 1907, también la aconsejaron. La siguieron muchos, a veces sin necesidad. Se mantuvo hasta la llegada de los antibióticos. A veces dejaban completamente abiertas las vejigas por miedo a las infecciones. (6)

En 1939 se le ocurrió a Gauthier la prostatectomía en tres tiempos y afortunadamente no tuvo seguidores. (10)

\section{Duval}

Realizó el primer intento de sutura hemostática en la celda prostática. Ejecutaba una técnica que consistía en suturar el cuello vesical con el muñón uretral. Cerraba por completo la vejiga y dejaba solo una sonda uretral. Por la dificultad en su realización y por el gran riesgo que existía en aquella época de la aparición de infecciones, a consecuencia de cerrar pronto la vejiga, tuvo malos resultados y la abandonó. $(10,45)$

Cincuenta y ocho años más tarde Puigvert y colaboradores adoptaron a la vía transvesical, un procedimiento parecido de sutura hemostática que era

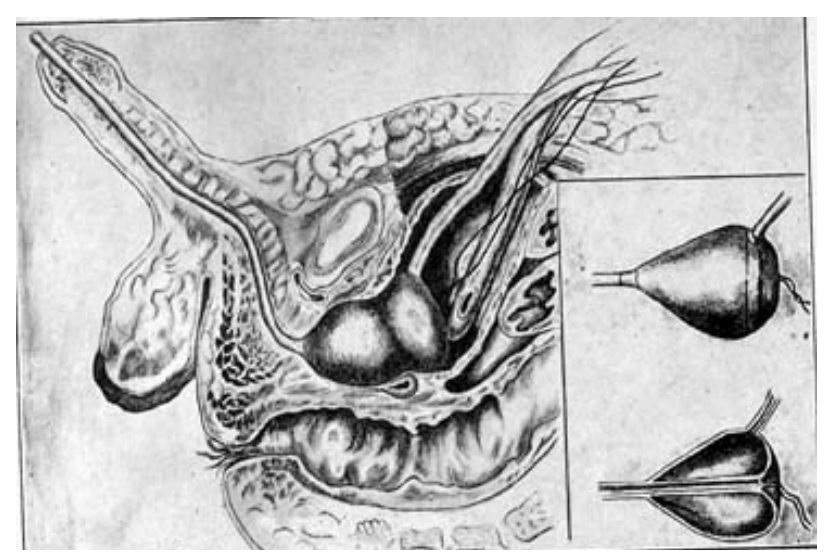

FIG URA 10. Sonda balón de Pilcher. 
propia de la operación perineal: anastomosis términoterminal vésico-uretral, pero por esta vía los puntos sólo los podía dar en la zona posterior del cuello. (49)

\section{León Cardenal}

La técnica de Freyer se extendió por toda Europa. La primera adenomectomía realizada en Madrid, la llevó a cabo el Dr. Cardenal, que era cirujano general del Hospital de la Princesa. (9)

\section{Young}

Ideó un instrumento llamado por él, "Extirpador Tubular Prostático". Era una especie de cuchilla endouretral tubular (the punch) Con las modificaciones de Braasch, Bumpus y Caulk se le incorporó la eléctrica para realizar hemostasia. (5)

\section{Van Stockum}

Fue el primero en realizar una prostatectomía retropúbica extravesical. Le siguieron Hildebrant en 1912, Lidski en 1922, y 0 tto Maier en 1924. Pero el gran difusor de esta técnica fue Millin, muchos años después. (59)

\section{Pasteau y Bensa}

Por este mismo año estos autores idean un dedil metálico en forma de uña afilada para favorecer el inicio de la enucleación del "adenoma". Era más eficaz que

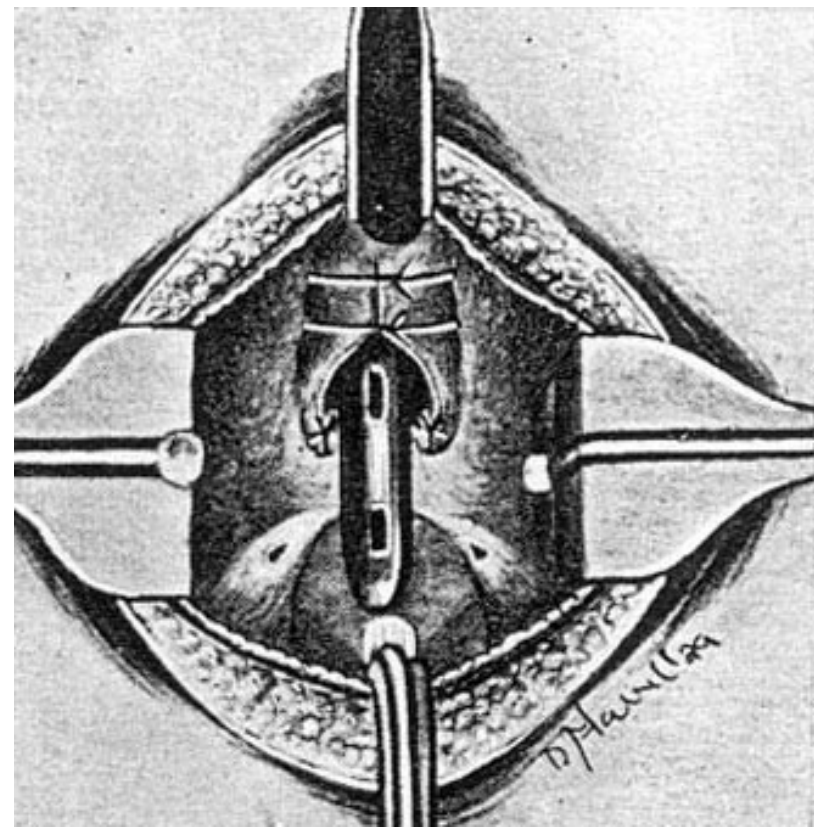

FIG URA 11. Técnica de Harris. la uña del cirujano, como solía hacer Freyer. También servía para cortar la uretra al final de la enucleación. 0 tros la iniciaban con bisturí, con tijeras o rompiendo el puente anterior uretral entre los dos lóbulos laterales. $(6,45,58)$

\section{Freyer}

Ideó el taponamiento con gasas de la celda prostática para combatir las frecuentes hemorragias postoperatorias. Escat, tiempo después, colocaba gasas yodofórmicas, con un hilo por la uretra y otro por arriba para retirarlas a los 3-5 días. Colocaba, después, una sonda uretral que retiraba a los 6-8 días.

Marion en 1930 había realizado 2820 adenomectomías con taponamiento de la celda en la mayoría de los casos. (10)

\section{Hagner}

Construyó una bolsa de goma para cohibir la hemorragia, que colocaba dentro de la celda prostática. Era casi igual que la de James Briggs diseñada en 1906. Pilcher la modificó en 1917. El mismo fin tiene la del español Sicre colocada en el recto. En 1937 Foley diseña su célebre sonda que posteriormente fue modificada con esta finalidad (sonda balón de Delinotte). $(10,51)$ (Figura 10)

\section{Thomson-Walker}

Propugna la operación "abierta", para poder ver y actuar en la zona operatoria, en lugar de la "cerrada" realizada hasta entonces. Daba algunos puntos de hemostasia con gran rapidez. Diseñó un separador vesical con una lámpara incorporada. $(45,54)$

\section{Ramón Camiña}

En el VI Congreso Nacional de Urología, la primera ponencia fue escrita por él: Cistoscopia Hipogástrica". En ella opinaba lo siguiente: "O s diré lo que pienso de la operación de Freyer... es la operación urológica menos reglada en el sentido absoluto de la palabra... que después de ejecutada deja al cirujano en mayor incertidumbre... ¿Será este caso de los bien operados que se mueren o de los mal operados que se salvan?..."

\section{Stern}

Dota al cistoscopio de Nite (1880) de un dispositivo eléctrico con un asa metálica para resecar tejidos. Poco después Mac Carthy lo perfeccionó. Al principio sólo realizaban un canal estrecho que luego, al mejo- 
rar la técnica, fue ampliandose. Barnes y Nesbit en 1943 describen detalladamente esta técnica.

Este mismo año Beer utiliza por vez primera la electrocirugía en urología. $(5,50,54)$

\section{Lower (USA)}

Después de la enucleación empezó a realizar una sutura de la celda sobre la sonda, con excelente efecto hemostático, por lo que la mayoría de las veces podía cerrar primariamente la vejiga. Luego era necesario hacer dilataciones cérvico-uretrales para normalizar el flujo urinario. $(37,39)$

\section{Harris (A ustralia)}

El mismo año a este cirujano australiano, se le ocurrió una técnica muy parecida: Daba puntos en el cuello vesical a la altura de 5 y 7 , y otro en el centro del borde posterior. Luego, otros transversales sobre la sonda. $(27,29,37)$ (Figura 11)

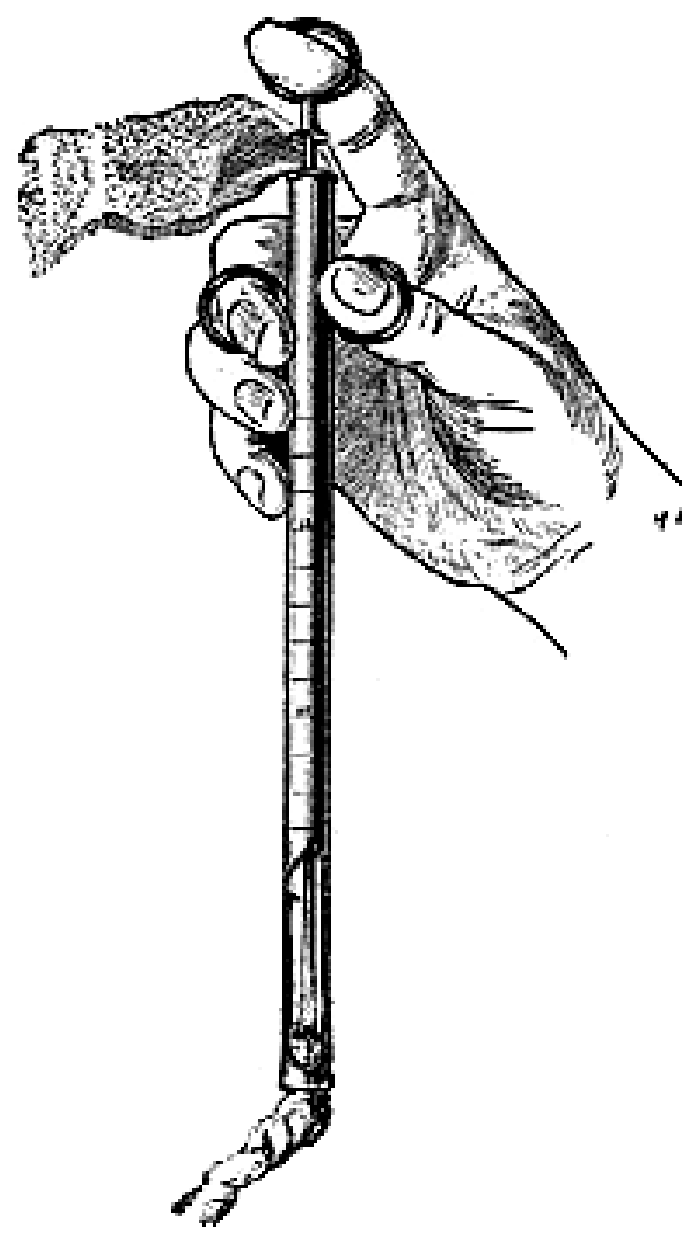

FIG URA 12. Tubo de G erota para el taponamiento de la celda prostática.

\section{Legueu}

Estimaba que el 5-6\% de los enfermos que se operaban, morían por la anestesia general. Por ello prefería la anestesia local con novocaína. Ideó varias clases de agujas para la infiltración de la pared abdominal, pared anterior de vejiga, cavidad vesical, región prostática, cuello vesical y la uretra prostática. Realizaba la ligadura de los deferentes previamente a la incisión de la vejiga, para disminuir la incidencia de orquiepididimitis. La incisión vesical la hacía alta y pequeña. El dedo en el recto lo consideró muy importante. Hacía taponamiento de la celda cuando era fácil la enucleación, pues según él eran los más sangrantes. Si era necesario hacía la operación en dos tiempos. (38)

Treinta años más tarde Ellis usó la anestesia local pero acompañada de anestesia espinal baja. (20)

\section{Marion}

Fue un gran entusiasta del taponamiento de la celda prostática. Con el tubo de taponamiento de Gerota se facilitaban las maniobras. Hasta el 20 día no comenzaba los lavados de la vejiga y ya retiraba parcialmente las mechas. El tubo lo iba cambiando por otros más pequeños. A partir del 60 día añadía lavados por uretra. Al 100 retiraba el último tubo y esperaba 2-3 días para colocar la sonda uretral. El enfermo curaba en 20-25 días. No recomendaba levantarlo pronto (media de 18 días). (45) (Figura12)

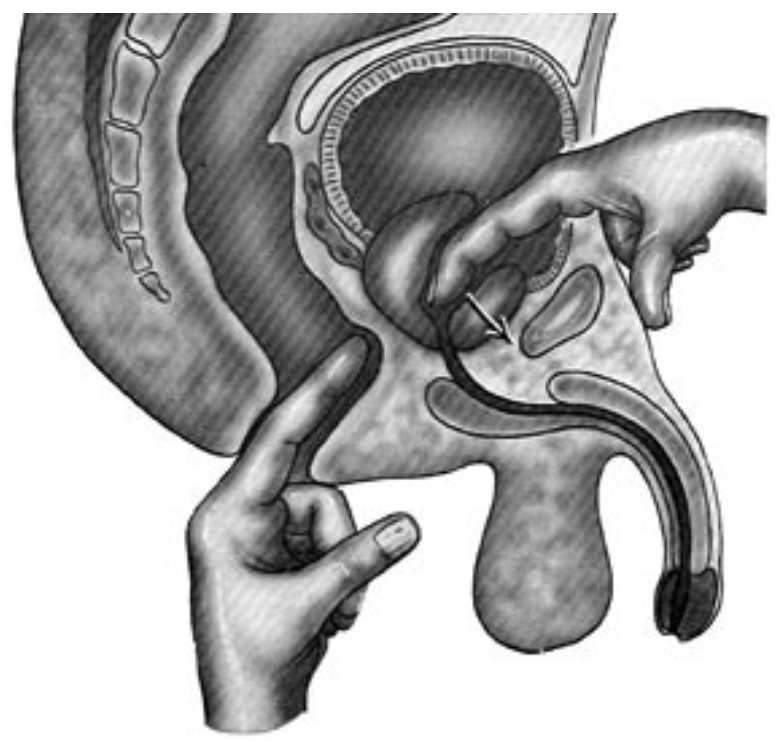

FIG URA 13. Rotura del "puente" entre los lóbulos laterales. 


\section{Jacobs y Cooper}

Practicaron la incisión prostática vertical, sin llegar a vejiga.

En 1935 Hybbinette comunicó un método creado por su compatriota sueco Henrickson que consistía en abrir cápsula prostática y vejiga a la vez. En 1948 Ward y en 1954 Bourque, perfeccionaron la técnica. Con esta apertura hacían mejor hemostasia y resolvían patología vesical añadida. Leabetter la modificó en 1959. $(5,7,37)$

\section{Mc Carthy}

A ntes de cerrar la vejiga se le ocurrió la electrocoagulación por vía transuretral de los puntos sangrantes y la resección de irregularidades que hubiera. Le secundaron Davis en 1948 y Hyman, Leiter y G lickman en 1951. En 1959, Hutch realizaba esta técnica inmediatamente después de haber cerrado la vejiga. En 1955 la empleó Gorostiago en cuatro casos de prostatectomía perineal introduciendo el resectoscopio a través de la incisión del periné. $(32,33)$

\section{Millin}

Era un gran reseccionista, pero su fama se debe al perfeccionamiento de la vía retropúbica extravesical. O pinaba que: "... ninguna de las vías clásicas hasta ahora en uso posee las características de una verdadera intervención ideal..." (44)

Esto que opinaba de las vías de acceso más usadas y posiblemente el no poder realizar resecciones transuretrales, fue el motivo de que perfeccionara la antigua técnica de Von Stockum. Durante la II Guerra Mundial los bisturís eléctricos más poderosos fueron expropiados de las clínicas y usados para bloquear el sistema enemigo de radar. Ésta técnica con su buena exposición, limpieza, hemostasia y simplicidad, fue una gran revelación para los urólogos "formados en el hedor y la suciedad de la vieja operación suprapúbica con su gran tubo suprapúbico, la bolsa prostática y el resto de los espantosos aparatos “ (5)

Es una cirugía que proporciona muy buena visibilidad y permite hacer una excelente hemostasia. El enfermo sufre menos molestias y esta menos días hospitalizado. (43)

\section{Ockerblad}

Enucleó uno de los "adenomas" más grandes hasta entonces: pesó 820 gramos. En España, en 1987, el doctor Montañés extirpó uno de 810 gramos. $(42,45)$

\section{Hryntschak (Austria)}

Técnica de prostatectomía suprapúbica con cierre primario de la vejiga. Simplificó la técnica de Harris, del que la aprendió en 1935. No daba el punto en el borde posterior, sino que lo cortaba en cuña. A parecían estenosis cérvico-uretrales muy a menudo. $(2-4,29)$

\section{Henry}

Sugirió la ligadura previa de las arterias prostáticas para controlar la hemorragia postoperatoria. Lo denominó "Ligadura vascular preliminar". Jain realizaba en 1968 la ligadura de las arterias próstatovesicales y de los plexos venosos prostáticos.

En 1989 Walsh y O esterlich recomiendan una técnica muy parecida. $(34,60)$

\section{Muller-Meernach}

Practicó la ligadura de la arteria iliaca interna después de la adenomectomía. (45) Una medida muy drástica que se encuentra en el polo opuesto de la actitud de algunos urólogos, que opinaban que la principal hemostasia se realizaba por la contracción del cuello y de la cápsula prostática. Las maniobras y suturas a esta altura dificultarían este mecanismo natural. $(16,24)$

\section{Deisting}

Ideó un aparato para la divulsión de la próstata. Realizaba con él una dilatación forzada de la uretra prostática rompiendo la comisura anterior y posterior. Sus seguidores no encontraron tan buenos resultados como él preconizaba (1)

\section{Boeminghaus}

Recomienda la introducción del dedo índice de la mano izquierda en el recto para elevar la próstata; la rotura digital del puente anterior para iniciar la enucleación, y para cerrar la vejiga, la sutura en bolsa de tabaco añadiendo un punto de seguridad en forma de zeta. $(6,58)$ (Figura 13)

\section{Harvard y White}

Describen una técnica suprapúbica con incisión vesical transversal baja. Con ella se conseguía gran visibilidad y por lo tanto mejor hemostasia. Le siguen otros autores como 0 'Conor. $(28,48)$ 

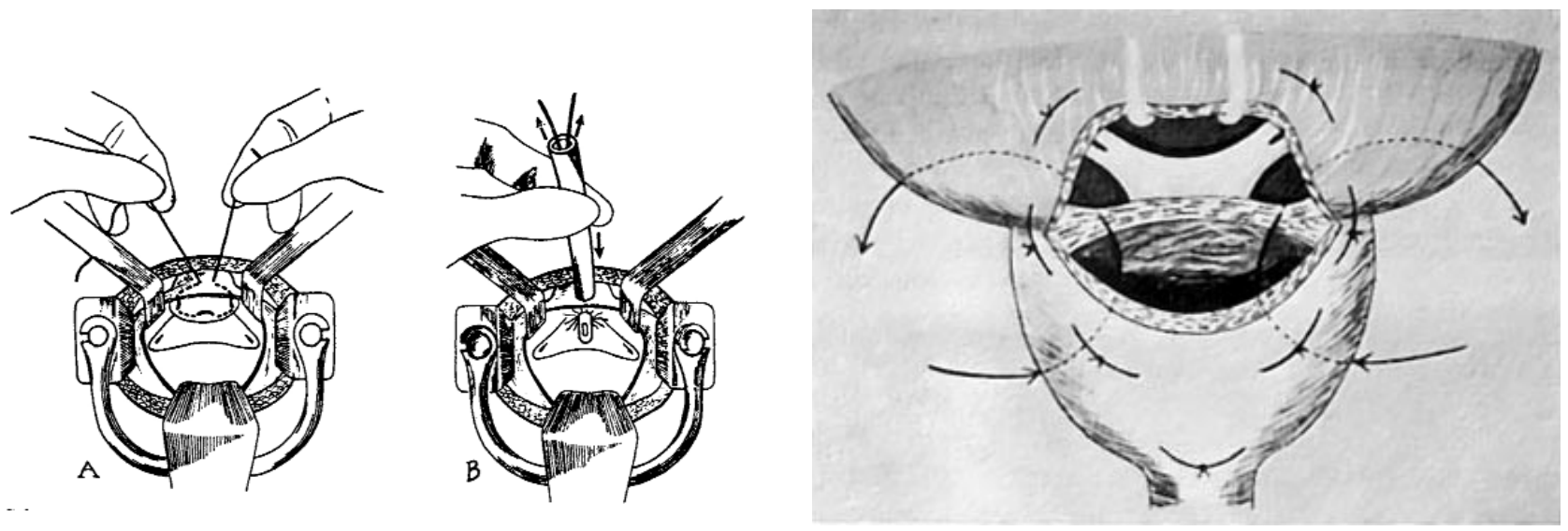

FIG URA 14. Técnica de Alfonso de la Peña.

FIG URA 15. Técnica de G regoir.

\section{Alfonso de la Peña}

Ideó una nueva técnica para realizar hemostasia después de la adenomectomía. Realizaba cerclaje del

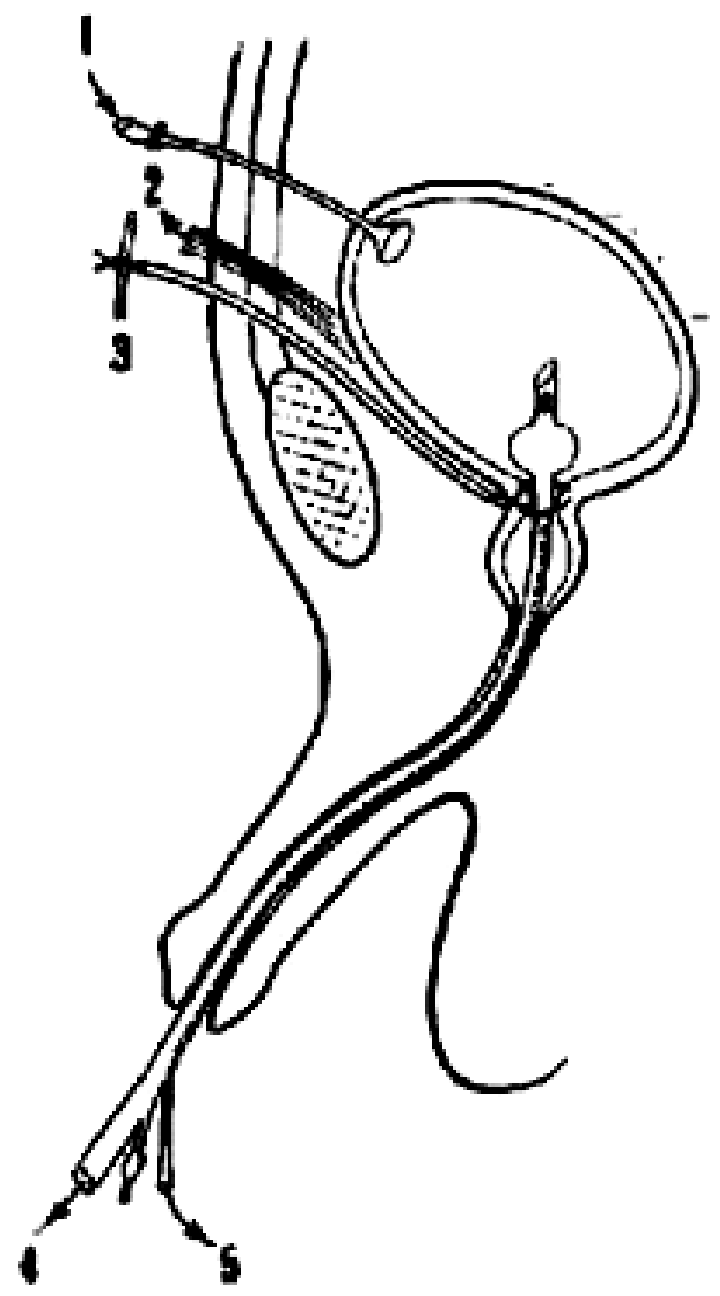

FIG URA 16. Técnica de Denis-Churet cuello con una sutura continua en bolsa de tabaco que exteriorizaba por hipogastrio. El cerclaje lo retiraba a los 2-3 días. No había peligro de estenosis.

Tres años después Malament popularizó en el mundo anglosajón la misma técnica y Jouvar en los Países del Este de Europa, en 1968. (15, 41, 55) (Figura 14)

\section{Gregoir}

Ideó la "Adenomectomía Hemostática". Daba puntos antes y después de la enucleación. Luego trigonizaba la uretra posterior con puntos separados. Los resultados eran muy variables. $(25,43)$ (Figura 15$)$

\section{Denis}

Basada en la técnica de Alfonso de la Peña, ideó la "A denomectomía del Vacío". Con una sonda especial, solamente aspiraba el contenido de la celda prostática después de hacer el cerclaje del cuello.

Más tarde, Churet añadió un globo a la sonda y desde entonces es conocida como técnica de Denis-Churet. $(17,19)$ (Figura 16)

\section{Hubmer}

Realizó 350 operaciones sin colocar sonda uretral. Esta técnica ya la había realizado Couvelaire en 1955. $(10,31)$

\section{0'Conor}

Describe una técnica de plicatura de la celda prostática con muy buenos resultados hemostáticos. Se podía realizar tanto con la vía suprapúbica como con la retropúbica. (47) 


\section{BIBUOGRAFIA y LECTURAS RECOMENDADAS (*lectura de interés y $* *$ lectura fundamental)}

1. ABOULKER, P.; STEG, A.: "La divulsion de la prostate d'apres observations personalles". J. D’Urol. et Nephrol., 70:337, 1964.

2. AlCAlÁ SANTAELlA, R.: "La Prostatectomía de Hryntschak y el valor de la hemostasia". Arch. Esp. Urol., 9: 129, 1953.

3. BECK, A.D.; GAUDIN, H.J.: "The Hryntschak prostatectomy: I. A review of 1346 cases". J. Urol., 103: $637,1970$.

4. BECK, A.D.; GAUDIN, H.J.: "The Hryntschak prostatectomy.: II. A late review of 179 cases". J. Urol., 103: 778,1970

**5. BLANDY, J. P.: "Resección transuretral." Edit. Jims. Barcelona. 1981.

*6. BOEMINGHAUS, H.: "Urología. Terapéutica operatoria, clínica e indicaciones". Segunda Edición. Editorial Paz Montalvo. Madrid. 1958.

7. BOURQUE, J.P.: "Transvesicocapsular prostatic adenomectomy (transcommissural): preliminary report on 80 cases". J. Urol., 72:918, 1954.

**8. BUSH, R.B.; BUSH, I.M.: "Urologic Pathology: The Prostate". Tannenbaum, M. Lea\&Febiger. Philadelphia. 1977.

9. CARDENAL, D.L.: "Algo sobre el tratamiento de la hipertrofia de la próstata". Siglo Médico. 130, 1908.

**10. CIFUENTES DELATTE, L.: "Historia de la prostatectomía (un breve esquema evolutivo)". Comunicación a la Real Academia Nacional de Medicina el 22-1-1974. An. R. A. Med., XCI: 3, 1974.

**11. CIFUENTES DÍAZ, P.: "Bosquejo histórico de la urología en España anterior al siglo XIX”. Discurso leído en la solemne Sesión Inaugural celebrada el día 16 de marzo de 1945 en la Real Academia de Medicina". Imprenta de J. Cosano. Madrid. 1945.

**12. DEAVER, J.B.: "Enlargement of the prostate". Ed. P. Blakiston's Son \& Co. Philadelphia. 1905.

13. DE LA PEÑA, A.: "Resección transuretral. Historia y técnica" Rev. Esp. de Urol.. 3: 173, 1975.

*14. DE LA PEÑA, A.: "Epistemología Urológica. Evolución de un saber". Discurso leído en la Real Academia de Medicina para la recepción publica del académico electo Excmo. Sr. D. Alfonso de la Peña. Diciembre de 1968.

15. DE LA PEÑA, A.; ALCINA, E.: "Suprapubic Prostatectomy: a new technique to prevent bleeding". J. Urol., 88: 86, 1962.

16. DE LA PEÑA, L.; MERINO, G.: "La adenomectomía transvesical anhemostática o sangrante". Arch. Esp. Urol., 18: 106, 1965.

17. DE PONCE SOCORRO, J.M.: "Adenomectomía prostática de Dennis-Churet. Aportación de seis casos y comentarios sobre la técnica". Act. Urol. Esp., 7: 3, 1983.

*18. DÍAZ, F.: "Tratado nuevamente impreso de todas las enfermedades de los riñones, vejiga y carnosidades de la verga, y urina". Editado por la Real Academia Nacional de Medicina, con un estudio preliminar acerca del autor y sus obras, por el Dr. Rafael Mollá.
Biblioteca Clásica de la Medicina Española. Imprenta de Julio Cosano. Madrid. 1923.

19. DUQUE ARANA, J.I.: "Adenomectomía bajo depresión. Nuestra experiencia y revisión de la literatura". Arch. Esp. Urol., 41: 108, 1988.

20. ELLIS, H.; LEATHERDALE, R.A.L.: "Prostatectomy; anaesthetic technique and other factors affecting prognosis". Lancet, 2: 1189, 1958.

*21. FREYER, P.J.: "A clinical lecture on total extirpation of the prostate for radical cure of enlargement of that organ: with four successful cases". Br. Med. J., ii: 125, 1901.

*22. FREYER, P.J.: "Total enucleation of the prostate in advanced old age". Lancet, 25: 490, 1905.

*23. FULLER, E.: "The question of priority in the adoption of the method of total enucleation, suprapubically, of the hypertrophied prostate". Ann. Surg., 41: 520, 1905.

24. GOODYEAR, W.A.; BEARD, D.E.: "Blood loss in Prostatectomy". J. Urol., 62: 849, 1949.

25. GREGOIR, W.: "L'Adénomectomie Hemostatíque". Acta. Urol. Belg., 36: 337, 1968.

26. GUIARD, F.P.: "Le Traitement du Prostatisme (Hypertrophie de la Prostate) a l'usage des praticiens". Octave Doin, Editeur. Paris. 1901.

*27 HARRIS, S.H.: "Suprapubic Prostatectomy with closure". Br. J. Urol., 1: 285, 1929.

28. HARVARD, B.M.; WHITE, R.R.: "A technic for transvesical Prostatectomy”. Conn. Med., 24: 286, 1960.

*29. HRYNTSCHAK, T.: "Suprapubic transvesical prostatectomy with primary closure of the bladder". J. Int. Coll. Surg., 15:366, 1951.

30. HUARD, P.; IMBAULT-HUART, M-J.: "Andrés Vesalio. Iconografía Anatómica". Ed. TEMIS. Prom. Med. Farm. Lab. Beecham. Barcelona, 1983.

31. HUBMER, G.; LIPSKY, H.; PETRITSCH, y cols: “ Prostatectomy with a No-catheter technique". Br. J. Urol., 49: 315, 1977.

32. HUTCH, J.A.: "Combined Prostatectomy". J. Urol., 83: 67, 1960.

33. HYMAN, A.; LEITER, H.E.; GLICKMAN, S.L.: "Suprapubic prostatectomv with hemostasis by transurethral fulguration and primary closure of the bladder". J. Mt. Sinai Hosp., 17: 652, 1951.

34. JAIN, A.C.: "A new method of prostatectomy with preliminary ligation of blood vessel". Acta. Urol. Belg., 36: 349, 1968.

*35. LAGUNA, A.: "Methodus cognoscendi extirpandique excrescentes in vesicae collo carunculas, 1551". Facsímil editado por la Oficina de Historia de la Asociación Española de Urología. Madrid. 2004.

36. LAIÍN ENTRALGO, P.: "Historia universal de la medicina”. Primera edición. Salvat Editores, S.A.. Barcelona. 1972.

37. LEADBETTER, G.W.; DUXBERRV, J.H.; LEADBETTER, W.F.: "Can prostatectomy be improved?". J. Urol., 82: 600, 1959.

*38. LEGUEU, F.: "Tratado médico quirúrgico de las vías urinarias". Segunda edición. Salvat Editores S.A.Barcelona. 1927.

39. LOWER, W.E.: "Cierre completo de la vejiga después de la prostatectomía. Informe preliminar". J.A.M.A.E.E., 18: 343, 1927.

**40. MAGANTO, E.; CLEMENTE, L.; CARRERA, C.: 
"La prostatectomía: una técnica quirúrgica en continua evolución". En Hitos en la Historia de la Urología. Pulso Editorial S.A.1999.

41. MALAMENT, M.: "Maximal hemostasis in suprapubic Prostatectomy”. Surg. Gynecol. Obstet., 120: 1307, 1965.

42. MARTIN VIVALDI, J.: "Estudio comparativo de la prostatectomía retropubiana y la transvesical tipo Hryntschak". Arch. Esp. Urol., 13: 68, 1957.

43. MARTÍNEZ PIÑEIRO, J.A.; HERNÁNDEZ, A.; CORRAL, F.J. y cols: "La adenomectomía retropúbica". Arch. Esp. Urol. 23: 23, 1970.

*44. MILLIN, T.: "Retropubic prostatectomy. A new extravesical technique. Report on 20 cases". Lancet. ii: 693,1945 .

**45. MURPHY, L.J.T.: "The History of Urology". Edit. Charles C. Thomas. Springfield, 1972.

46. NICOLL, G.A.; RIFFLE, G.; ANDERSEN, F.: "Suprapubic Prostatectomy the removable purse: a continuing comparable analysis of 300 consecutive cases". J. Urol., 120: 702, 1978.

47. O'CONOR, V.J.JR.: "An aid for hemostasis in open prostatectomy: capsular plication”. J. Urol., 127: 448, 1982.

48. O' CONOR, V.J.JR.; BULKLEY, C.J.; SOKOL, J.K.: "Low suprapubic prostatectomy: Comparison of results with the standard operation in two comparable groups of 142 patients". J. Urol. 90: 301, 1963.

49. PUIGVERT, A.; MARTÍNEZ, A.: "Prostatectomía transvesical hemostática". Arch. Esp. Urol., 12: 185, 1964.

*50. PURPON, I.: "Prostatectomía transuretral endoscópica". Queromón Ed. S. A. Madrid 1979.

51. RATHOD, D.M. y cols.: "Comparison of Pilcher bag technique with standard suprapubic Prostatectomy". Urology. Vol. XV. 4:1039. 1980.

**52. RAYMOND, G.; CHEVALIER, D.; AMIEL, J.: "1987: Centième anniversaire de l'adenomectomie prostatique transvesicale”. J. D`Urol., 94: 853. 1988.

**53. RIERA PALMERO, J.: "La urología española del siglo XVIII". Cuadernos de Historia de la Medicina. IV. 1: $55,1965$.

**54. SHACKLEY, D.: "Un siglo de Cirugía prostática". Brit. J. Urol. International., 83: 776, 1999.

55. SHEVTSOV, I.P.; GORIACHEV, I.A.: "Urología Operatoria”. Editorial Mir Moscú. Moscú. 1988.

56. STUTZMAN, R.E.: "Open Prostatectomy". En Urologic Surgery. Glenn, J. F. Fourth edition. J. B. Lippincott Company. Philadelfia. 1991.

*57. TILlaUX, P.: “Tratado de Cirugía Clínica”. Segunda edición. Biblioteca Ilustrada de Espasa y Ca $\mathrm{C}^{\mathrm{a}}$ Editores. Barcelona. 1885.

58. VALVERDE MAZUELAS, A.: "La prostatectomía transvesical con cierre primitivo y completo de vejiga”. Arch. Esp. Urol., 10: 248, 1954.

*59. VAN STOCKUM, W.J.: "Prostatectomía suprapúbica extravesicalis". Zentr. Chir,: 2: 41, 1909.

60. WALSH, P.C.; OESTERLING, J.E.: "Improved hemostasis during simple retropubic prostatectomy", J. Urol., 143: 1203, 1990. 